\title{
Comparison of dust charging between Orbital-Motion-Limited theory and Particle-In-Cell simulations
}

\author{
Gian Luca Delzannd* and Xian-Zhu Tangt \\ Theoretical Division, Los Alamos National Laboratory, Los Alamos, NM 87545
}

(Dated: October 5, 2018)

\begin{abstract}
The Orbital-Motion-Limited (OML) theory has been modified to predict the dust charge and the results were contrasted with the Whipple approximation [Tang and Delzanno, Phys. Plasmas 21, 123708 (2014)]. To further establish its regime of applicability, in this paper the OML predictions (for a non-electron-emitting, spherical dust grain at rest in a collisionless, unmagnetized plasma) are compared with Particle-In-Cell simulations that retain the absorption radius effect. It is found that for large dust grain radius $r_{d}$ relative to the plasma Debye length $\lambda_{D}$, the revised OML theory remains a very good approximation as, for the parameters considered $\left(r_{d} / \lambda_{D} \leq 10\right.$, equal electron and ion temperatures), it yields the dust charge to within $20 \%$ accuracy. This is a substantial improvement over the Whipple approximation. The dust collected currents and energy fluxes, which remain the same in the revised and standard OML theories, are accurate to within $15-30 \%$.
\end{abstract}

PACS numbers: 52.25.Dg, 52.27.Lw, 52.65.-y

\footnotetext{
* delzanno@lanl.gov

$\dagger$ xtang@lanl.gov
} 


\section{INTRODUCTION}

The Orbital-Motion-Limited (OML) theory [1 13$]$ is the most widely used dust charging theory. In its simplest form (i.e. without dust electron-emission processes), it neglects barriers to ion current collection that are created by local maxima of the effective potential (the so-called absorption radius concept [4]). This approximation results in simple expressions for the collected currents and energy fluxes to the grain, and allows to calculate the steady-state dust potential without solving Poisson's equation.

Until recently OML was known to be unable to calculate the plasma response to the charging process, that is, the OML Poisson equation could not be solved even numerically. This was pointed out by Allen et al. [4], who used the OML plasma densities calculated in Ref. [2] to show that OML is intrinsically inconsistent. In particular, they showed that OML is valid when the shielding potential decreases more slowly than $1 / r^{2}$, but that in a Maxwellian plasma this condition is always violated in the quasi-neutral plasma region away from the dust grain (for any dust grain size, at least if the electron temperature is greater or equal than the ion temperature). Allen et al. [4] attributed this inconsistency to the absorption radius effect, which is missing in OML since the effective potential is assumed to be monotonic.

These limitations imply that OML could not self-consistently calculate the dust charge, a quantity of fundamental importance for dust transport studies since it controls the magnitude of the electromagnetic force: the dust charge depends on how the charged dust is screened by the plasma, namely on how the plasma rearranges around the dust grain in response to the charging process, and requires the full solution of Poisson's equation. In practice, this has restricted the successful application of OML to cases where the dust radius is much smaller than the plasma Debye length, where one can accurately obtain the dust charge from the dust potential by either neglecting screening effects or assuming that these

effects can be described by a Debye-Huckel potential near the grain with a given screening length.

In order to assess in very simple terms the importance of screening effects, one can start from Gauss' law relating the dust charge $Q_{d}$ to the radial electric field at the grain surface $E_{r}$ (spherical symmetry is assumed where $r$ is the radial coordinate):

$$
Q_{d}=\left.4 \pi \varepsilon_{0} r_{d}^{2} E_{r}\right|_{r=r_{d}}
$$


( $\varepsilon_{0}$ is vacuum permittivity and $r_{d}$ is the dust radius). A standard Debye shielding calculation allows one to express $E_{r}=-d \phi / d r$ in terms of the Debye-Huckel electrostatic potential $\phi$ given by

$$
\phi=\phi_{d} \frac{r_{d}}{r} \exp \left(-\frac{r-r_{d}}{\lambda_{\text {lin }}}\right),
$$

where $\phi_{d}=\phi\left(r_{d}\right)$ is the dust potential and the screening length corresponds to the linearized Debye length

$$
\frac{1}{\lambda_{\text {lin }}^{2}}=\frac{1}{\lambda_{D e}^{2}}+\frac{1}{\lambda_{D i}^{2}},
$$

with the electron (ion) Debye length given by $\lambda_{D e, i}=\sqrt{\varepsilon_{0} T_{e, i} / e^{2} / n_{\infty}}$ (subscripts 'e' and 'i' label electrons and ions, respectively, $e$ is the elementary charge, $T_{e, i}$ are the electron and ion temperatures expressed in $\mathrm{eV}, n_{\infty}$ is the unperturbed plasma density and we have assumed singly charged ions). It follows that

$$
Q_{d}=4 \pi \varepsilon_{0} r_{d}\left(1+\frac{r_{d}}{\lambda_{\text {lin }}}\right) \phi_{d}
$$

which is referred to as the Whipple approximation [5]. Obviously, in the limit $r_{d} / \lambda_{\text {lin }} \ll 1$ Eq. (4) simply recovers Coulomb's law applied to a charged dust particle in vacuum,

$$
Q_{d}=4 \pi \varepsilon_{0} r_{d} \phi_{d}
$$

where one recognizes the familiar dust capacitance $C_{d}=4 \pi \varepsilon_{0} r_{d}$. Equation (41) indicates that when the dust grain radius becomes comparable to or larger than the screening length, plasma screening effects cannot be neglected and might in fact be dominant in the calculation of the dust charge. One should also keep in mind that in a fully self-consistent calculation both the dust potential and the screening length will depend on the dust radius, making the relation between the dust charge and the dust radius even more non-linear [6].

While the small dust-radius-to-Debye-length limit works well for many dusty plasma applications [7-9], important emerging applications can be in the opposite limit. For instance, this is the case of magnetic fusion energy applications [10], where the size of dust particles emitted from the tokamak walls can easily be comparable to or larger than the local Debye length [11, 12]. This is also true for dust particles injected on purpose [12 16]. As we will show in this paper, neglecting screening effects in this regime can lead to an order of magnitude underestimation of the dust charge. This could have important consequences for dust particles moving in the magnetized sheath near the tokamak walls, where the dynamics 
perpendicular to the wall is governed by the balance between electrostatic and drag forces [17, 18] and for a negatively charged particles a stronger electrostatic force would mean a higher probability of escape, wider excursions away from the wall and stronger heat fluxes [18]. In general, however, it is worth pointing out that it appears that most of the dust transport codes for magnetic fusion energy applications [13, 19 23] (which are all based on OML) use Eq. (5) to relate the dust charge to the dust potential, i.e. neglect screening effects.

Recently Tang and Delzanno [24] showed that the problems with the classic OML theory arise from a mistake in the integration limits used in the derivation of the ion density. Upon correcting this error, a revised OML theory that can now calculate the plasma response and, consequently, the dust charge [24, 25]. This theoretical development opens up the possibility to perform a true comparison between the OML theory and a Particle-In-Cell (PIC) approach that calculates the charging of a dust grain in a plasma from first principles (i.e. without invoking the OML approximation), and assess the validity/accuracy of OML in the large dust-radius-to-Debye-length limit. This is the goal of the present paper.

We note that this study is conducted with the most basic form of OML and it is the first necessary step towards properly accounting for screening effects. Other effects such as the presence of an ion flow, electron emission processes from the dust grain and magnetic field effects on current collection can be very important in magnetic fusion energy applications and their impact on dust charging and screening will have to be evaluated. Some of these effects do break the spherical symmetry on which OML rests, and require an upgraded theory together with fully self-consistent simulations. Whether some form of the OML theory that accounts for these non-OML effects in a simplified but reasonable way can still be formulated is, at this point, an open question.

The paper is organized as follows. In Sec. II we briefly describe the PIC simulations and the revised OML theory. In Sec. III we present a comparison between the two in terms of average dust charge, potential, collected currents and energy fluxes, and the plasma densities and electrostatic potential. In Sec. IV] we draw the conclusions of this study. 


\section{PARTICLE-IN-CELL SIMULATIONS AND REVISED OML THEORY}

\section{A. Particle-In-Cell simulations}

In this subsection we describe the PIC simulations of the charging and shielding of a dust grain in a plasma whose steady state is compared with that obtained by the revised OML theory.

The PIC simulations are conducted in spherical geometry and in the collisionless, electrostatic limit (i.e. the plasma particles move only in response to the self-consistent electric field generated in the system), with the same code used for instance in Refs. [26 29]. The spherical dust grain of radius $r_{d}$ is at rest in the center of the system and its surface corresponds to the inner boundary of the simulation domain. The outer boundary is a concentric sphere of radius $R$. At the outer boundary the system is open and we inject a Maxwellian plasma at every time step, to compensate for the plasma particles that leave the system due to their thermal motion. Moreover, the electrostatic potential is set to zero at the outer boundary. Particles that hit the dust grain are removed from the simulation and their charge is accumulated on the dust grain. This translates into a boundary condition on the electric field given by Gauss' law (we assume a perfectly conducting dust grain).

At time $t=0$ we load a Maxwellian plasma with temperature $T_{e}=T_{i}$, density $n_{e}=n_{i}=$ $n_{\infty}$ and the ion-to-electron mass ratio $m_{i} / m_{e}\left(\lambda_{D}=\lambda_{D e}=\lambda_{D i}\right)$. We choose $m_{i} / m_{e}=1836$. For the other simulation parameters, we use a grid with uniform radial spacing and $N_{r}=$ 1000 grid points, while the time step is $\omega_{p e} \Delta t=0.05\left[\omega_{p e}=\sqrt{e^{2} n_{\infty} /\left(\varepsilon_{0} m_{e}\right)}\right.$ is the electron

plasma frequency]. The electron thermal velocity is defined as $v_{t h, e}=\sqrt{T_{e} / m_{e}}$. We conduct a parametric study of dust charging varying the dust radius in the range $r_{d} / \lambda_{D}=0.25-10$. The outer domain boundary is at $R / \lambda_{D}=30$ for all the runs except when $r_{d} / \lambda_{D}=10$, where we use $R / \lambda_{D}=60$ (and $N_{r}=2000$ to keep the same level of resolution). The final time of the simulations is $\omega_{p e} T \sim 2000$ for $r_{d} / \lambda_{D} \geq 1$ and $\omega_{p e} T \sim 4000$ for $r_{d} / \lambda_{D}<1$. At the end of the simulations the average number of particles per cell is $\sim 3500-4000$ for $r_{d} / \lambda_{D} \geq 1$ and $\sim 7000$ for $r_{d} / \lambda_{D}<1$.

Initially the electrons charge the grain negatively because of their higher mobility. This creates a sheath electric field that eventually equilibrates the electron $\left(I_{e}\right)$ and ion $\left(I_{i}\right)$ currents to the grain and a steady state is reached when $I_{e}+I_{i}=0$ (floating condition) with 
the grain negatively charged. In the results presented in the next section, the steady-state data from PIC simulations is averaged over the last quarter of the simulations (labeled as $\left.T_{\text {ave }}\right)$, corresponding to $\omega_{p e} T_{\text {ave }} \sim 500$ for $r_{d} / \lambda_{D} \geq 1$ and $\omega_{p e} T_{a v e} \sim 1000$ for $r_{d} / \lambda_{D}<1$.

\section{B. Revised OML theory}

The steady state calculated from the revised OML theory amounts to solving Poisson's equation in spherical geometry

$$
\nabla^{2} \phi=\frac{e}{\varepsilon_{0}}\left(n_{e}^{O M L}-n_{i}^{O M L}\right)
$$

with the following expressions for the ion density [24, 25]

$$
\begin{aligned}
\frac{n_{i}^{O M L}(z)}{n_{\infty}}= & \sqrt{-\frac{\beta \varphi}{\pi}}\left[1+\sqrt{1-\frac{\varphi_{d}^{O M L}}{z^{2} \varphi}}\right]+\frac{e^{-\beta \varphi}}{2}[1-\operatorname{Erf}(\sqrt{-\beta \varphi})]+ \\
& \frac{\sqrt{1-z^{-2}}}{2} e^{-\beta \tilde{\varphi}}[1-\operatorname{Erf}(\sqrt{-\beta \tilde{\varphi}})], \quad \phi(z)<\frac{\phi_{d}^{O M L}}{z^{2}} ; \\
\frac{n_{i}^{O M L}(z)}{n_{\infty}}= & \sqrt{-\frac{\beta \varphi}{\pi}}+\frac{e^{-\beta \varphi}}{2}[1-\operatorname{Erf}(\sqrt{-\beta \varphi})]+\frac{\sqrt{1-z^{-2}}}{2} e^{-\beta \tilde{\varphi}}, \quad \phi(z) \geq \frac{\phi_{d}^{O M L}}{z^{2}},
\end{aligned}
$$

and electron density

$$
\begin{aligned}
\frac{n_{e}^{O M L}(z)}{n_{\infty}}= & \frac{1}{2}\left\{1+\operatorname{Erf}\left(\sqrt{\varphi-\varphi_{d}^{O M L}}\right)+\sqrt{1-z^{-2}}\left[1-\operatorname{Erf}\left(\sqrt{\frac{\varphi-\varphi_{d}^{O M L}}{1-z^{-2}}}\right)\right] \exp \left[\frac{\varphi-\varphi_{d}^{O M L}}{z^{2}-1}\right]\right\} \\
& \exp (\varphi) .
\end{aligned}
$$

In Eqs. (17), (8) and (9) we have defined the following quantities: $z=r / r_{d}, \varphi=e \phi / T_{e}$, $\beta=T_{e} / T_{i}, \tilde{\varphi}=\left(\varphi-\varphi_{d}^{O M L} / z^{2}\right) /\left(1-z^{-2}\right)$ and the dust potential is $\phi_{d}^{O M L}=\phi\left(r_{d}\right)$. Poisson's equation (6) is solved with the following boundary conditions: at the outer boundary we have

$$
\left.\frac{d \phi}{d r}\right|_{r=R}=-\frac{2}{R} \phi(R),
$$

which mimics the asymptotic behavior of the shielding potential $\phi \sim$ const $/ r^{2}$ [and can be easily verified in the quasi-neutral region by Taylor expanding Eqs. (8) and (9) in the limit $z \gg 1$ and $\varphi \ll 1$ ], while the floating potential condition is used on the dust grain

$$
I_{e}\left(\phi_{d}^{O M L}\right)+I_{i}\left(\phi_{d}^{O M L}\right)=0
$$


with currents given by

$$
\begin{aligned}
I_{i} & =e 4 \pi r_{d}^{2} n_{\infty} \sqrt{\frac{T_{i}}{2 \pi m_{i}}}\left(1-\frac{e \phi_{d}^{O M L}}{T_{i}}\right), \\
I_{e} & =-e 4 \pi r_{d}^{2} n_{\infty} \sqrt{\frac{T_{e}}{2 \pi m_{e}}} \exp \left(\frac{e \phi_{d}^{O M L}}{T_{e}}\right) .
\end{aligned}
$$

The dust charge is obtained by the solution of Poisson's equation via Gauss' law (11). Furthermore, the OML power collected by the grain from the plasma is obtained by [30]

$$
\begin{aligned}
& q_{i}=T_{i}\left(\frac{2-\frac{e \phi_{d}^{O M L}}{T_{i}}}{1-\frac{e \phi_{d}^{O M L}}{T_{i}}}-\frac{e \phi_{d}^{O M L}}{T_{i}}\right) \frac{I_{i}}{e} \\
& q_{e}=2 T_{e} \frac{\left|I_{e}\right|}{e} .
\end{aligned}
$$

While in the remainder of the text we will generally refer to the revised OML theory for the comparison with PIC, we note that the floating condition (11) in the revised OML theory has not changed relative to the standard OML theory and therefore the two theories produce the same value of floating potential, dust currents and energy fluxes. We reiterate that in the revised OML theory one can now solve Poisson's equation to get the screening near the grain and calculate the dust charge, which in general could not be done in the standard OML theory because of the inconsistencies discussed by Allen et al. [4].

For the discussion of the results of the next section, Eq. (6) has been solved numerically with a second-order accurate finite difference scheme. We introduce a relative error defined as

$$
\delta \varepsilon_{f}=\frac{\left|f^{P I C}-f^{O M L}\right|}{\left|f^{P I C}\right|},
$$

where $f$ represents a generic quantity of interest. Here and in the remainder of the paper we will use superscripts 'PIC' and 'OML' where necessary to indicate the values of $f$ obtained from PIC simulations or from the revised OML theory.

\section{RESULTS AND DISCUSSION}

Table I shows results concerning the dust charge $Q_{d}$, the dust potential $\phi_{d}$ and the parameter

$$
\Gamma=\frac{Q_{d}}{4 \pi \varepsilon_{0} r_{d} \phi_{d}}-1,
$$


TABLE I. Summary of the PIC and revised OML simulations: dust charge and potential. The dust potential obtained from OML is $\frac{e \phi_{d}^{O M L}}{T_{e}}=-2.5$.

\begin{tabular}{|c||c|c||c||c|c|}
\hline \hline$\frac{r_{d}}{\lambda_{D}}$ & $\frac{Q_{d}^{P I C}}{e n_{\infty} \lambda_{D}^{3}}$ & $\frac{Q_{d}^{O M L}}{e n_{\infty} \lambda_{D}^{3}}$ & $\frac{e \phi_{d}^{P I C}}{T_{e}}$ & $\Gamma^{P I C}$ & $\Gamma^{O M L}$ \\
\hline 0.25 & -10.00 & -9.87 & -2.51 & 0.27 & 0.25 \\
0.5 & -23.23 & -23.03 & -2.51 & 0.47 & 0.46 \\
1 & -57.64 & -57.46 & -2.54 & 0.81 & 0.83 \\
2 & -152.02 & -153.97 & -2.57 & 1.36 & 1.45 \\
3 & -277.39 & -286.24 & -2.60 & 1.83 & 2.03 \\
4 & -431.49 & -455.00 & -2.64 & 2.25 & 2.62 \\
5 & -612.52 & -661.41 & -2.68 & 2.63 & 3.20 \\
10 & -1898.75 & -2282.87 & -2.82 & 4.36 & 6.26 \\
\hline \hline
\end{tabular}

obtained from the PIC simulations and the revised OML theory. As expected, the dust charge increases with the dust radius. A least-squares fit of the data between $r_{d} / \lambda_{D}=3$ and $r_{d} / \lambda_{D}=10$ shows that $Q_{d} \propto r_{d}^{1.6}$, indicating that the non-linear corrections to Eq. (5), i.e. the screening from the plasma, is important. Moreover, there is a good agreement between the PIC simulations and the revised OML theory: up to $r_{d} / \lambda_{D} \sim 5, \delta \varepsilon_{Q}<10 \%$, and the biggest discrepancy obtained at $r_{d} / \lambda_{D}=10$ is only $\delta \varepsilon_{Q} \simeq 20 \%$. Most importantly, using the Whipple approximation (44) overestimates the dust charge by a factor of 2.5: for $r_{d} / \lambda_{D}=10, Q_{d} /\left(e n_{\infty} \lambda_{D}^{3}\right) \simeq-4757\left(\lambda_{\text {lin }} / \lambda_{D}=1 / \sqrt{2}\right)$. If one had used expression (5) (which is appropriate only when screening effects can be neglected), the dust charge would be grossly underestimated: for $r_{d} / \lambda_{D}=10, Q_{d} /\left(e n_{\infty} \lambda_{D}^{3}\right) \simeq-314$, a factor of 6 smaller than that obtained by PIC. Similar considerations can be drawn by looking at the dust potential. While the OML dust potential is independent of the dust radius, $\frac{e \phi_{d}^{O M L}}{T_{e}}=-2.5$, the PIC simulations show that the dust potential becomes more negative as the dust radius increases. These results are consistent with those of Ref. [31], and reflect the fact that as the dust radius grows, current collection becomes similar to that occurring in planar geometry rather than in spherical geometry (where plasma particles might miss the dust grain because of angular momentum effects and the related centrifugal force). The agreement between PIC and revised OML is very good up to $r_{d} / \lambda_{D} \sim 10$, with $\delta \varepsilon_{\phi}<12 \%$. 
TABLE II. Summary of the PIC simulations: dust currents. For the PIC simulations, the ion current is equal to the electron current since at steady state the floating condition $I_{e}^{P I C}+I_{i}^{P I C} \simeq 0$ is satisfied. Note that for the fourth and fifth columns the OML currents are calculated from expressions (12) and (13) but using the dust potential obtained from PIC (and shown in Table @) instead of $e \phi_{d}^{O M L} / T_{e}=-2.5$.

\begin{tabular}{|c||c|c|c|c||}
\hline \hline$\frac{r_{d}}{\lambda_{D}}$ & $\frac{I_{e}^{P I C}}{e n_{\infty} v_{t h, e} r_{d}^{2}}$ & $\frac{I_{e}^{P I C}}{I_{e}^{O M L}\left(\phi_{d}^{O M L}\right)}$ & $\frac{I_{e}^{P I C}}{I_{e}^{O M L}\left(\phi_{d}^{P I C}\right)}$ & $\frac{I_{i}^{P I C}}{I_{i}^{O M L}\left(\phi_{d}^{P I C}\right)}$ \\
\hline 0.25 & -0.40 & 0.97 & 0.98 & 0.97 \\
0.5 & -0.41 & 1.00 & 1.01 & 0.99 \\
1 & -0.40 & 0.96 & 1.00 & 0.96 \\
2 & -0.39 & 0.94 & 1.01 & 0.93 \\
3 & -0.37 & 0.90 & 1.00 & 0.88 \\
4 & -0.36 & 0.87 & 1.00 & 0.84 \\
5 & -0.34 & 0.83 & 1.00 & 0.80 \\
10 & -0.30 & 0.73 & 1.00 & 0.67 \\
\hline \hline
\end{tabular}

Table II shows the electron and ion currents collected by the dust grain at steady state, again varying the dust radius. Note that Table II only reports $I_{e}^{P I C}$ since at steady state the dust grain is at floating potential (although the floating condition is only satisfied in an average sense, $I_{e}^{P I C}+I_{i}^{P I C} \simeq 0$ ). One can see that the electron current, normalized to a reference current $I_{n o r m}=e n_{\infty} v_{t h, e} r_{d}^{2}$, decreases for larger dust radii, consistent with the fact that the dust potential is becoming more negative: for $r_{d} / \lambda_{D}=10, I_{e} / I_{\text {norm }}$ is about $27 \%$ less than that for $r_{d} / \lambda_{D} \lesssim 1$. Furthermore, Table II also presents the ratio of the collected plasma currents to those obtained from the OML theory using however the dust potential obtained by PIC simulations (Table【) instead of $e \phi_{d}^{O M L} / T_{e}=-2.5$. One can see that taking into account the change of the dust potential with $r_{d}$ results in an electron collected current in excellent agreement with expression (13). For the ions, on the other hand, this is true only for $r_{d} / \lambda_{D} \lesssim 1$ and there is a progressively larger departure from expression (12) as $r_{d}$ grows: for $r_{d} / \lambda_{D}=10, I_{i}^{P I C} / I_{i}^{O M L}\left(\phi_{d}^{P I C}\right)=0.68$.

Table III shows the electron $q_{e}^{P I C}$ and ion $q_{i}^{P I C}$ power collected by the dust grain obtained from the PIC simulations, with the same format of Tables \and II. As expected, the results (which are normalized to a reference power, $q_{\text {norm }}=T_{e} e n_{\infty} v_{t h, e} r_{d}^{2}$ ) are consistent with those 
TABLE III. Summary of the PIC simulations: power collected by the dust grain. Note that in the fourth and seventh columns the OML collected power is calculated using the dust potential obtained from PIC and shown in Table 【

\begin{tabular}{|c||c|c|c||c|c|c||}
\hline \hline$\frac{r_{d}}{\lambda_{D}}$ & $\frac{q_{e}^{P I C}}{T_{e} n_{\infty} v_{t h, e} r_{d}^{2}}$ & $\frac{q_{e}^{P I C}}{q_{e}^{O M L}\left(\phi_{d}^{O M L}\right)}$ & $\frac{q_{e}^{P I C}}{q_{e}^{O M L}\left(\phi_{d}^{P I C}\right)}$ & $\frac{q_{i}^{P I C}}{T_{e} n_{\infty} v_{t h, e} r_{d}^{2}}$ & $\frac{q_{i}^{P I C}}{q_{i}^{O M L}\left(\phi_{d}^{O M L}\right)}$ & $\frac{q_{i}^{P I C}}{q_{i}^{O M L}\left(\phi_{d}^{P I C}\right)}$ \\
\hline 0.25 & 0.79 & 0.96 & 0.97 & 1.49 & 0.96 & 0.96 \\
0.5 & 0.81 & 0.99 & 1.00 & 1.56 & 1.00 & 1.00 \\
1 & 0.79 & 0.96 & 1.00 & 1.53 & 0.99 & 0.96 \\
2 & 0.78 & 0.95 & 1.01 & 1.52 & 0.98 & 0.95 \\
3 & 0.74 & 0.90 & 1.00 & 1.49 & 0.96 & 0.91 \\
4 & 0.72 & 0.87 & 1.00 & 1.47 & 0.95 & 0.88 \\
5 & 0.69 & 0.83 & 1.00 & 1.44 & 0.93 & 0.84 \\
10 & 0.60 & 0.73 & 1.00 & 1.32 & 0.85 & 0.72 \\
\hline \hline
\end{tabular}

in Table II and show a variation of the dust collected power which is comparable to that of the dust collected currents.

Figure 1 shows the average plasma densities obtained from PIC (solid lines) and from the revised OML theory (dashed lines). In general, despite some standard PIC noise, there is very good agreement on the electron densities at least up to $r_{d} / \lambda_{D} \sim 5$, with more visible differences for $r_{d} / \lambda_{D}=10$. For the ion density, on the other hand, there is reasonable agreement between theory and simulations up to $r_{d} / \lambda_{D} \sim 1$. Although the PIC noise is still quite high for $r_{d} / \lambda_{D} \lesssim 1$, some non-monotonicity in the PIC ion density might be inferred near the dust grain, as in the revised OML theory. However, for $r_{d} / \lambda_{D}>2$, one can see that the PIC simulations show an ion density which increases monotonically with distance from the grain, typical of a sheath in planar geometry. The revised OML density, however, remains monotonically decreasing near the grain, as expected from a sheath in spherical geometry [6].

Figure 2 shows the average potential (normalized to the dust potential) in the simulation domain for various dust radii. Note that the potential for each simulation run is normalized to its respective floating potential (which depends on the dust radius, as shown in Table 【) to facilitate an assessment of the screening length. In general there is a very good agreement between theory and simulations at least up to $r_{d} / \lambda_{D} \sim 5$ and the screening from the plasma 
is captured correctly by the revised OML theory (hence the very good agreement on the dust charge already visible from Table (I).

\section{CONCLUSIONS}

In this paper we have presented a comparison between PIC simulations of the steady-state charging and shielding of a dust grain in a plasma and the revised OML theory. The latter was developed explicitly in Ref. 24] for the purpose of evaluating the plasma response to the charging process in the OML framework. This yields the dust charge including screening effects self-consistently.

We have performed a parametric study changing the dust radius in the regime of interest to magnetic fusion energy applications, i.e. when the dust grain is larger than the plasma Debye length. We have considered the simplest case of a non-electron-emitting spherical dust grain at rest in a collisionless, unmagnetized plasma. While the revised OML theory still cannot capture the transition from a spherical to a planar sheath that occurs when the dust becomes too large (as shown already in Ref. [31]), for the parameters considered (hydrogen plasma and $T_{e} / T_{i}=1$ ) we have shown that the revised OML theory remains a very good approximation up to $r_{d} / \lambda_{D} \sim 10$. In particular, the dust charge is accurate to within $20 \%$ once screening effects are correctly included. For comparison, for $r_{d} / \lambda_{D}=10$ the Whipple approximation overestimates the dust charge by a factor of 2.5 , while neglecting screening effects completely would underestimate the dust charge by almost one order of magnitude. Other quantities of interest to tokamak dust transport studies, like currents and energy fluxes collected by the dust from the plasma, remain the same in the revised and standard OML theories and are overestimated by only $15-30 \%$ for $r_{d} / \lambda_{D} \lesssim 10$. The contributions to the screening of other effects that can be important in magnetic fusion energy applications, like ion-flow, electron emission and magnetic fields, will be explored in the future.

\section{ACKNOWLEDGMENTS}

This research was supported by the U.S. Department of Energy Office of Science, Office of Fusion Energy Sciences, under the auspices of the National Nuclear Security Administration 
of the U.S. Department of Energy by Los Alamos National Laboratory, operated by Los Alamos National Security LLC under contract DE-AC52-06NA25396.

[1] H. Mott-Smth and I. Langmuir, Physical Review 28, 0727 (1926).

[2] Ya. L. Al'pert, A. V. Gurevich, and L. P. Pitaevskii, Space Physics with Artificial Satellites (Plenum Press, New York, 1965).

[3] J. Laframboise, Theory of spherical and cylindrical Langmuir probes in a collisionless, Maxwellian plasma at rest, Tech. Rep. (Toronto Univ. (Ontario). Inst. for Aerospace Studies, 1966).

[4] J. Allen, B. Annaratone, and U. de Angelis, Journal of Plasma Physics 63, 299 (2000).

[5] E. Whipple, Reports on progress in Physics 44, 1197 (1981).

[6] J. Daugherty, R. Porteous, M. D. Kilgore, and D. Graves, Journal of Applied Physics 72, 3934 (1992).

[7] P. K. Shukla and A. A. Mamun, Introduction to Dusty Plasma Physics (Institute of Physics Publishing, Philadelphia, 2001).

[8] V. E. Fortov and G. E. Morfill, Complex and Dusty Plasmas: From Laboratory to Space (CRC Press, Boca Raton, 2010).

[9] A. Bouchoule, Dusty Plasmas: Physics, Chemistry and Technological Impacts in Plasma Processing (Wiley, Chichester, 1999).

[10] S. I. Krasheninnikov, R. D. Smirnov, and D. L. Rudakov, Plasma Physics and Controlled Fusion 53, 083001 (2011).

[11] J. Sharpe, D. Petti, and H.-W. Bartels, Fusion Engineering and Design 6364, 153 (2002).

[12] D. Rudakov, A. Litnovsky, W. West, J. Yu, J. Boedo, B. Bray, S. Brezinsek, N. Brooks, M. Fenstermacher, M. Groth, E. Hollmann, A. Huber, A. Hyatt, S. Krasheninnikov, C. Lasnier, A. McLean, R. Moyer, A. Pigarov, V. Philipps, A. Pospieszczyk, R. Smirnov, J. Sharpe, W. Solomon, J. Watkins, and C. Wong, Nuclear Fusion 49, 085022 (2009).

[13] S. Ratynskaia, L. Vignitchouk, P. Tolias, I. Bykov, H. Bergsåker, A. Litnovsky, N. den Harder, and E. Lazzaro, Nuclear Fusion 53, 123002 (2013).

[14] A. Litnovsky, D. Rudakov, S. Bozhenkov, R. Smirnov, S. Ratynskaia, H. Bergsker, I. Bykov, N. Ashikawa, G. D. Temmerman, Y. Xu, S. Krasheninnikov, W. Biel, S. Brezinsek, J. Coenen, 
A. Kreter, M. Kantor, H. Lambertz, V. Philipps, A. Pospieszczyk, U. Samm, G. Sergienko, O. Schmitz, and H. Stoschus, Journal of Nuclear Materials 438, Supplement, S126 (2013), proceedings of the 20th International Conference on Plasma-Surface Interactions in Controlled Fusion Devices.

[15] S. Ratynskaia, P. Tolias, A. Shalpegin, L. Vignitchouk, M. D. Angeli, I. Bykov, K. Bystrov, S. Bardin, F. Brochard, D. Ripamonti, N. den Harder, and G. D. Temmerman, Journal of Nuclear Materials , (2014).

[16] X. Z. Tang and G. L. Delzanno, Journal of Fusion Energy 29, 407 (2010).

[17] S. I. Krasheninnikov, Y. Tomita, R. D. Smirnov, and R. K. Janev, Physics of Plasmas 11 (2004).

[18] G. L. Delzanno and X. Z. Tang, Physics of Plasmas 21, 022502 (2014).

[19] A. Pigarov, S. Krasheninnikov, T. Soboleva, and T. Rognlien, Physics of Plasmas 12, 122508 (2005).

[20] J. Martin, M. Bacharis, M. Coppins, G. Counsell, and J. Allen, EPL 83 (2008), 83/65001.

[21] M. Bacharis, M. Coppins, and J. E. Allen, Phys. Rev. E 82, 026403 (2010).

[22] R. D. Smirnov, A. Y. Pigarov, M. Rosenberg, S. I. Krasheninnikov, and D. A. Mendis, Plasma Physics and Controlled Fusion 49, 347 (2007).

[23] L. Vignitchouk, P. Tolias, and S. Ratynskaia, Plasma Physics and Controlled Fusion 56, 095005 (2014).

[24] X. Z. Tang and G. L. Delzanno, Physics of Plasmas 21, 123708 (2014).

[25] T. Bystrenko and A. Zagorodny, Physics Letters A 299, 383 (2002).

[26] G. L. Delzanno, G. Lapenta, and M. Rosenberg, Physical Review Letters 92, 350021 (2004).

[27] G. L. Delzanno, A. Bruno, G. Sorasio, and G. Lapenta, Physics of Plasmas 12, 062102 (2005).

[28] G. L. Delzanno, E. Camporeale, J. D. Moulton, J. E. Borovsky, E. A. MacDonald, and M. Thomsen, IEEE Transactions on Plasma Science 41, 3577 (2013).

[29] G. L. Delzanno and X. Z. Tang, Phys. Rev. Lett. 113, 035002 (2014).

[30] C. M. Ticos, Z. Wang, G. L. Delzanno, and G. Lapenta, Physics of Plasmas 13, 103501 (2006).

[31] C. T. N. Willis, M. Coppins, M. Bacharis, and J. E. Allen, Plasma Sources Science and Technology 19, 065022 (2010). 

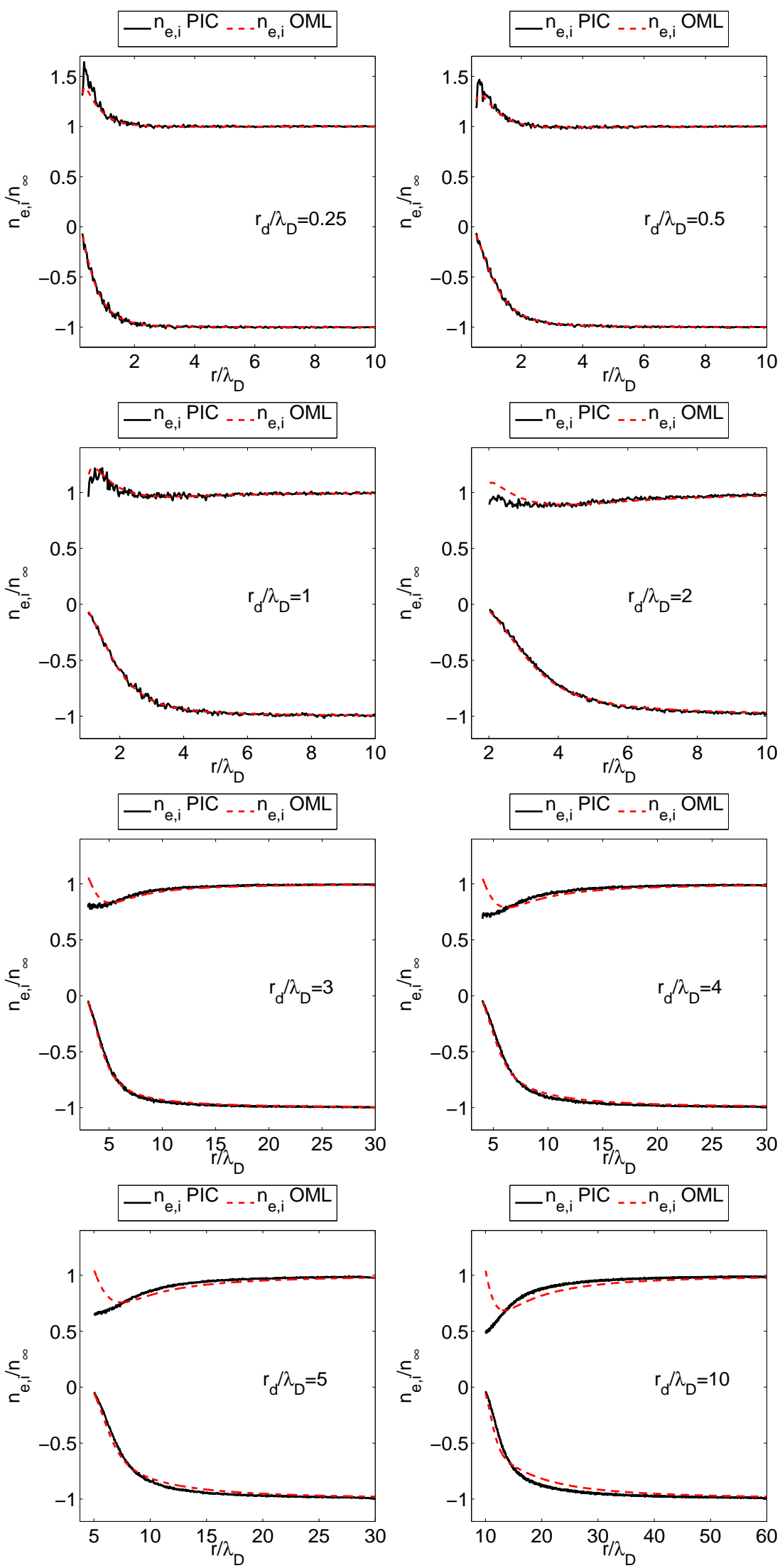

FIG. 1. Comparison of the steady-state plasma densities from PIC simulations (solid lines) and from the revised OML theory (dashed lines) varying the dust radius. For $r_{d} / \lambda_{D} \leq 2$ only part of the computational domain is shown. 

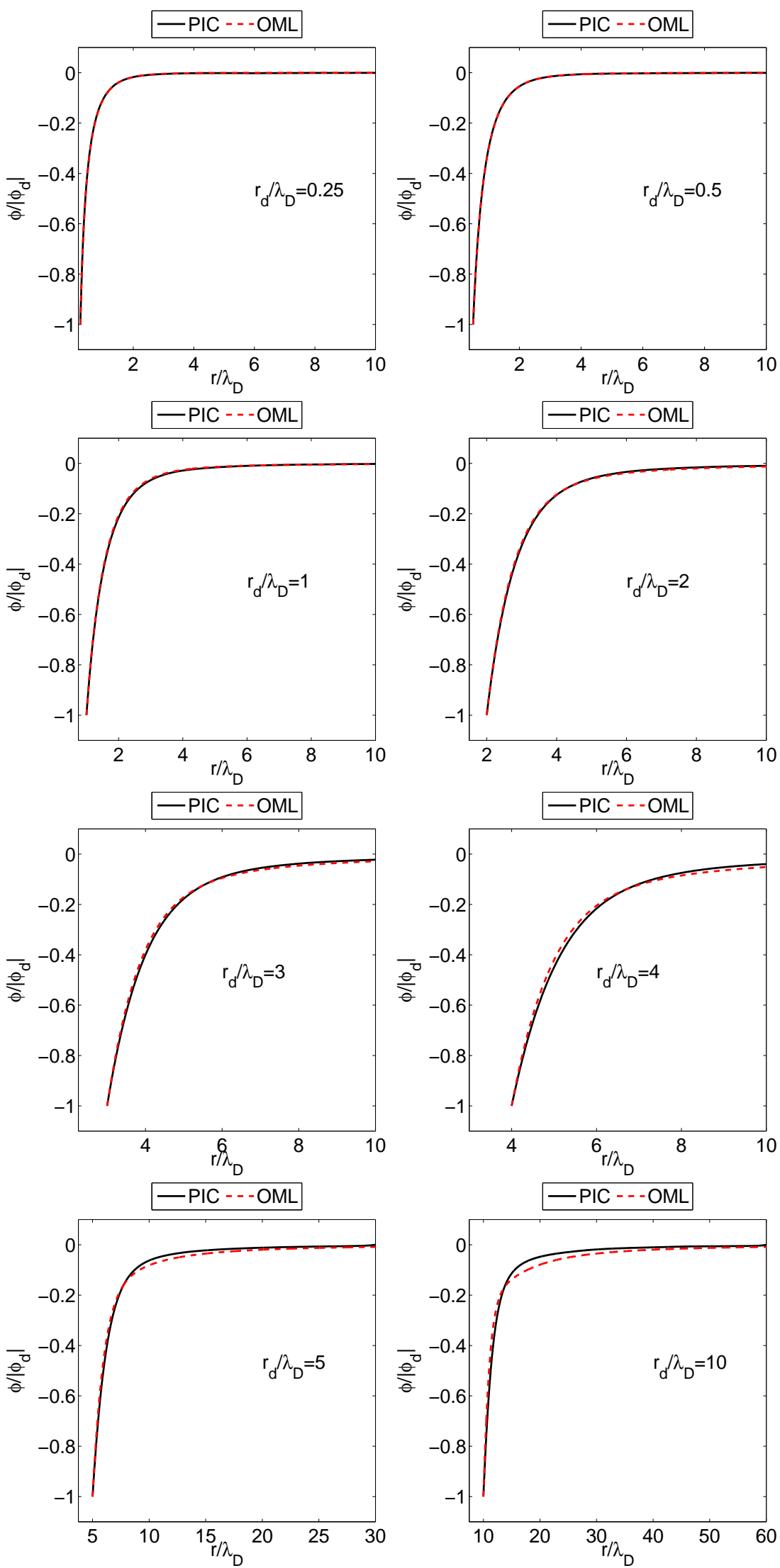

FIG. 2. Comparison of the steady-state electrostatic potential from PIC simulations (solid line) and from the revised OML theory (dashed line) 15 varying the dust radius. For $r_{d} / \lambda_{D} \leq 4$ only part of the computational domain is shown. 